at 6 months $(p=0.014)$. Median IBDQ bowel subset score improved from 41 at baseline to 50 at 6 months $(p<0.0005)$. Significant improvement was also found in the median VIO score from 11 at baseline to 8 at 6 months $(\mathrm{p}<0.0005)$. The median CTCAE rectum bowel mean score for men improved from 1.4 at baseline to 0.9 at 6 months and for women from 1.4 at baseline to 1.3 at 6 months. Pooling male and female data, the CTCAE mean score significantly improved comparing baseline with 6 month scores $(p=0.001)$.

Conclusion GI symptom questionnaire scores significantly improved from baseline to 6 months. This suggests that structured gastroenterological evaluation using an algorithmic approach may improve GI symptoms in this patient group, although a controlled study is necessary to confirm this.

Competing interests None declared.

\section{PTU-148 DOES INVESTIGATING CHRONIC GASTROINTESTINAL SYMPTOMS FOLLOWING PELVIC RADIOTHERAPY IDENTIFY TREATABLE DIAGNOSES?}

doi:10.1136/gutjnl-2012-302514c.148

${ }^{1} \mathrm{C}$ C Henson, ${ }^{*} \mathrm{~J}$ McLaughlin, ${ }^{2} \mathrm{Y}$ Ang, ${ }^{3} \mathrm{C}$ Babbs, ${ }^{4} \mathrm{~J}$ Crampton, ${ }^{4} \mathrm{M}$ Kelly, ${ }^{3} \mathrm{~S}$ Lal, ${ }^{5} \mathrm{~J}$ Limdi, ${ }^{6} \mathrm{G}$ Whatley, ${ }^{1} \mathrm{R}$ Swindell, 'W Makin, 'S E Davidson. ' Christie Hospital, Manchester, UK; ${ }^{2}$ University of Manchester, Manchester, UK; ${ }^{3}$ Salford Royal Foundation Trust, Salford, UK; ${ }^{4}$ University Hospital of South Manchester NHS Trust, Manchester, UK; ${ }^{5}$ Pennine Acute Hospitals NHS Trust, Manchester, UK; ${ }^{6}$ Tameside Hospital NHS Foundation Trust, Ashton under Lyne, UK

Introduction 17000 patients are treated with radical pelvic radiotherapy per year in the UK. Although 50\% develop significant chronic gastrointestinal (GI) symptoms, $<20 \%$ are referred for gastroenterological evaluation. We aimed to determine the causes of GI symptoms in this patient group.

Methods 60 patients with GI symptoms $\geq 6$ months after radical pelvic radiotherapy were identified from oncology clinics. Those requiring urgent investigation via the 2 -week wait pathway were excluded. Baseline characteristics including demographic data, cancer treatment details and symptoms were collected. Patients were referred for gastroenterological evaluation using an algorithmic approach, which involves the identification of all GI symptoms and investigation for all potential causes for the individual symptoms. Details of investigations and diagnoses were collected.

Results 20 men and 36 women with primary gynaecological (31), urological (17) or lower GI (8) tumours were included, with a median age of 58.5 years (range 26.9-81.8). As part of their cancer treatment 15 patients also had brachytherapy, 28 had chemotherapy and 25 had surgery. Patients presented with multiple GI symptoms (median 8, range 4-16) including frequency (46), urgency (52), loose stool (50), faecal incontinence (40), flatulence (43), bloating/ distension (38) and rectal bleeding (29). The median number of investigations per patient was 9 (range 1-17), including routine blood tests (47), coeliac screen (39), breath tests for small bowel bacterial overgrowth (21) and lactose intolerance (16), SeHCAT scans (27) and upper (27) and lower (38) GI endoscopy. Common diagnoses include radiation proctopathy (22) and bile acid mabsorption (12). Some diagnoses are unrelated to previous radiotherapy, for example, diverticulosis (9) and colonic polyps (8). No cause was found for symptoms in seven patients. 25 patients have 2 or more GI diagnoses.

Conclusion Gastroenterological evaluation identifies significant and potentially treatable diagnoses in patients who develop chronic GI symptoms following pelvic radiotherapy. Some findings are incidental and some are unrelated to previous cancer treatment. GI symptoms in these patients have historically been considered "untreatable". These data suggest that structured gastro- enterological assessment has the potential to improve outcome by identifying these diagnoses and facilitating focussed treatment.

Competing interests None declared.

\section{PTU-149 CAMBRIDGE-MIAMI RISK ASSESSMENT FOR INTESTINAL TRANSPLANTATION}

doi:10.1136/gutjnl-2012-302514c.149

${ }^{1} \mathrm{C}$ Pither, ${ }^{2} \mathrm{R}$ Sivaprakasam, ${ }^{3} \mathrm{H}$ Takahashi, ${ }^{3} \mathrm{~S}$ Nishida, ${ }^{2} \mathrm{~A}$ Butler, ${ }^{3} \mathrm{~J}$ Moon ${ }^{1} \mathrm{M} D$ Dawwas, ${ }^{4} \mathrm{~S}$ Gabe, ${ }^{2} \mathrm{~N}$ Jamieson, ${ }^{5} \mathrm{~J}$ Woodward, ${ }^{6} \mathrm{E}$ Island, ${ }^{6} \mathrm{~A}$ Tzakis, ${ }^{1} \mathrm{~S}$ J Middleton. ${ }^{1}$ Gastroenterology, Cambridge University, Cambridge, UK; ${ }^{2}$ Transplantation Surgery, Cambridge University, Cambridge, UK; ${ }^{3}$ Transplantation Surgery, Department of Surgery, University of Miami School of Medicine, Miami, USA ${ }^{4}$ Intestinal Failure Unit, St Mark's Academic Centre, London, UK; ${ }^{5}$ Gastoenterology, Cambridge University, Cambridge, UK; ${ }^{6}$ Transplantation Surgery, Department of Surgery, University of Miami School of Medicine, Miami, USA

Introduction The Cambridge-Miami (CaMi) preoperative risk assessment score has been previously validated in a small cohort and accurately predicted the survival after intestinal transplantation. We undertook a further validation in a larger cohort of patients.

Methods Co-morbidity and lost venous access are used as putative preoperative risk factors, each scored $0-3$ for severity. Patients (72 adults (M:F, 33:39) received an isolated intesinal graft (27), or a cluster graft including intestine (45).

Results Mean (SD) survival was 1501 (1444) days. The Kaplan-Meier analysis of survival revealed a significant inverse association between survival and CaMi score [logrank test for trend, $\mathrm{p}<0.0001]$. Patients were grouped into CaMi scores of 0 and 1,2 and 3,4 and 5, 6 and above, and HR [95\% CIs] for death (compared to group $0+1$ ) was found to increase as the CaMi score increased; 1.945 [0.7622 to 5.816], 5.075 [3.314 to 36.17] and 13.77 [463.3 to 120100] respectively and was significantly greater than group $0+1$ at group 4 $+5(\mathrm{p}<0.0001)$.

Conclusion The ability to predict survival from the CaMi score might allow better patient selection, and identify patients for earlier transplantation.

Competing interests None declared.

\section{PTU-150 QUALITY OF LIFE BEFORE AND AFTER INTESTINAL TRANSPLANTATION}

doi:10.1136/gutjnl-2012-302514c.150

${ }^{1} \mathrm{C}$ Pither, ${ }^{*}{ }^{1} \mathrm{~S}$ Duncan, ${ }^{1} \mathrm{H}$ Tincknell, ${ }^{1} \mathrm{C}$ Hanson, ${ }^{1} \mathrm{~B}$ Chukualim, ${ }^{1} \mathrm{~J}$ Woodward ${ }^{2} \mathrm{~A}$ Butler, ${ }^{1} \mathrm{~S}$ Middleton. ${ }^{1}$ Gastroenterology, Cambridge University Hospitals NHS Foundation Trust, Cambridge, UK; ${ }^{2}$ Transplant Surgery, Cambridge University Hospitals NHS Foundation Trust, Cambridge, UK

Introduction Survival following intestinal transplantation has substantially improved over the last decade and if this trend continues quality of life (OOL) may be considered as a major indication for transplantation. It is important to establish if QOL can be enhanced by transplantation and whether some aspects are more inclined to improve than others.

Methods QOL was assessed using Short form 36 (SF36) in a cohort of consecutive patients who had either been assessed for, undergone or, were awaiting transplantation. Data were scored using validated criteria for different QOL functions. The statistical package SPSS (IBM) was used to analyse the data.

Results 62 data sets were available, 26 pre-transplant and 36 posttransplant. Grouped data showed significantly better physical function $\left(p=0.03^{*}\right)$, social functioning $\left(p=0.01^{*}\right)$, general health $\left(p=0.006^{*}\right)$ and emotional role limitation $(p=0.02 *)$ in the 